\title{
Identification of Differentially Methylated miRNA Genes During Compatible and Incompatible Interactions Between Soybean and Soybean Cyst Nematode
}

\author{
Aditi Rambani, ${ }^{1}$ Yanfeng Hu, ${ }^{1,2}$ Sarbottam Piya, ${ }^{1}$ Miao Long, ${ }^{1}$ J. Hollis Rice, ${ }^{1}$ Vince Pantalone, ${ }^{1}$ and \\ Tarek Hewezi ${ }^{1+}$ \\ ${ }^{1}$ Department of Plant Sciences, University of Tennessee, Knoxville, TN 37996, U.S.A. \\ ${ }^{2}$ Key Laboratory of Mollisols Agroecology, Northeast Institute of Geography and Agroecology, Chinese Academy of Sciences, \\ Harbin, China
}

Accepted 5 August 2020.

\begin{abstract}
DNA methylation is a widespread epigenetic mark that affects gene expression and transposon mobility during plant development and stress responses. However, the role of DNA methylation in regulating the expression of microRNA (miRNA) genes remains largely unexplored. Here, we analyzed DNA methylation changes of miRNA genes using a pair of soybean (Glycine max) near-isogenic lines (NILs) differing in their response to soybean cyst nematode (SCN; Heterodera glycines). Differences in global DNA methylation levels over miRNA genes in response to SCN infection were observed between the isogenic lines. miRNA genes with significant changes in DNA methylation levels in the promoter and primary transcript-coding regions were detected in both lines. In the susceptible isogenic line (NIL-S), 82 differentially methylated miRNAs were identified in response to $\mathrm{SCN}$ infection whereas, in the resistant isogenic line (NIL-R), only 16 differentially methylated miRNAs were identified. Interestingly, gma-miR5032, gma-miR5043, gma-miR1520b, and gma-2107ch16 showed opposite methylation patterns in the isogenic lines. In addition, the miRNA paralogs gma-miR5770a and gmamiR5770b showed hypermethylation and hypomethylation in NIL-S and NIL-R, respectively. Gene expression quantification of gma-miR5032, gma-miR5043, gma-miR1520b, and gmamiR5770a/b and their confirmed targets indicated a role of DNA methylation in regulating miRNA expression and, thus, their targets upon SCN infection. Furthermore, overexpression of these four miRNAs in NIL-S using transgenic hairy root system enhanced plant resistance to SCN to various degrees with a key role observed for miR5032. Together, our results provide new insights into the role of epigenetic mechanisms in controlling miRNA regulatory function during $\mathrm{SCN}$-soybean interactions.
\end{abstract}

\section{A. Rambani and Y. Hu contributed equally to this work. \\ ${ }^{\dagger}$ Corresponding author: T. Hewezi; thewezi@utk.edu}

Funding: This work was funded by the Tennessee Soybean Promotion Board and the University of Tennessee Institute of Agriculture to the Hewezi laboratory.

*The $\boldsymbol{e}$-Xtra logo stands for "electronic extra" and indicates that supplementary figures and supplementary tables are published online.

The author(s) declare no conflict of interest.

(c) (1) () $\odot$ Copyright $\odot 2020$ The Author(s). This is an open access article (C) (1) () () distributed under the CC BY-NC-ND 4.0 International license.
Keywords: DNA methylation, gene expression, isogenic lines, miRNA, nematode-plant interactions, soybean, soybean cyst nematode

Methylation of cytosine residues in DNA is a well-defined epigenetic mark that affects gene expression, transposon mobility, and genome integrity (He et al. 2011). In the plant genome, methylated cytosines occur in three different contexts ( $\mathrm{CG}, \mathrm{CHG}$, and $\mathrm{CHH}$ ), where $\mathrm{H}$ denotes any base other than guanine $(\mathrm{G})$. After the advent of a bisulfite sequencing method that allows the detection of methylated cytosines with high precision at genome scale, the importance of this epigenetic mark in various aspects of gene expression and genome organization and evolution was realized (Choi et al. 2020; Pei et al. 2019). The regulatory function of cytosine methylation is context and region dependent (Feng et al. 2010; Suzuki and Bird 2008). For example, DNA methylation of gene promoters can affect gene expression by affecting the recruitment of regulatory proteins. Also, DNA methylation of transposable elements (TEs) can affect their mobility and the expression of their adjacent genes (Dowen et al. 2012; Wang et al. 2013). Furthermore, DNA methylation of gene body regions can influence gene transcription and alternative splicing (Attwood et al. 2002; Choi et al. 2020; Shayevitch et al. 2018).

Environmental stress factors are known to induce transient nonheritable changes in DNA methylation patterns (Boyko et al. 2010; Secco et al. 2015; Wibowo et al. 2016; YongVillalobos et al. 2015), which can alter gene expression levels at a global scale to activate adaptive response. Also, plant pathogens have been shown to induce dramatic changes in plant methylomes (Hewezi el al. 2018). Methylome changes induced by phytopathogens share a common feature of DNA hypomethylation patterns, which were found to affect specific genes involved in various biological processes and plant defense (Hewezi et al. 2018). However, microRNA (miRNA) genes have been frequently ignored in methylome analysis. The observation that miRNA genes are tightly controlled and frequently respond to pathogen infection raises the possibility of miRNAs being epigenetically modified by DNA methylation.

miRNAs are a class of noncoding small RNAs that play central roles in the regulation of gene expression at mostly posttranscriptional level. miRNAs are 21 to 22 nucleotides long and formed by processing of long primary transcripts (primiRNAs) folded into hairpin loop-like structures (Rogers and Chen 2013). The miRNA genes are transcribed by RNA 
polymerase II (Pol II) and the primary transcript is stabilized by addition of poly A tail to the 3' end. The hairpin-like structures of the pri-miRNAs are recognized by Dicer-like RNase III endonuclease 1 (DCL1) and processed into miRNA/miRNA* duplex. The miRNA/miRNA* duplex is methylated by HUA ENHANCER (HEN1) and exported to cytoplasm (Yu et al. 2005). In the cytoplasm, the functional strand of the mature miRNA is loaded together with Argonaute 1 (AGO1) protein into the RNA-induced silencing complex (RISC) to target mRNA transcripts containing perfect or near-perfect complementary sequence for degradation or translational repression, causing posttranscriptional gene silencing (Bartel 2004).

In animal systems, it has been shown that DNA methylation of miRNA genes affects their biogenesis and expression and that methylation over miRNA genomic regions is enhanced in stress- and disease-related phenotypes (Glaich et al. 2019; Han et al. 2007; Ju et al. 2020; Mo et al. 2019; Shen et al. 2012; Varghese et al. 2018). In plants, recent reports have also established the biological significance of DNA methylation of miRNAs. For example, Song et al. (2015) reported a negative correlation between DNA methylation at the $5^{\prime}$ flanking region and miRNA expression level during bisexual flower development in andromonoecious poplar (Populus tomentosa). This led to the identification of key miRNA genes regulated by DNA methylation during flower development. In addition, in $P$. simonii, various abiotic stress treatments were found to induce DNA methylation changes of miRNA genes with substantial influence on their abundance (Ci et al. 2015; Song et al. 2016). However, no similar study has been conducted thus far in Arabidopsis.

Plant-parasitic cyst nematodes (Heterodera spp.) are obligate biotrophs that infect the root system of host plants and establish a permeant feeding site, called the syncytium, from which the nematode feeds and completes its life cycle. Recent studies have revealed that soybean cyst nematodes ( $\mathrm{SCN}$; Heterodera glycines) and beet cyst nematodes (BCN; H. schachtii) induce DNA methylation changes in significant numbers of proteincoding genes and TEs in a context-specific fashion (Hewezi et al. 2017; Rambani et al. 2015, 2020). Also, it has been recently shown that the root-knot nematode Meloidogyne graminicola triggers hypomethylation in early-developing galls formed on rice roots (Atighi et al. 2020). Functional analysis of differentially methylated genes revealed their biological importance in plant-nematode interactions (Hewezi et al. 2017; Rambani et al. 2020). For example, Arabidopsis T-DNA insertional mutants of differentially methylated genes, including NRPDIa, Glu synthase 2, fatty acid reductase 4 , and $\beta$-galactosidase3, was found to enhance plant susceptibility to BCN (Hewezi et al. 2017). Also, overexpression of a receptor-like protein kinase and a dirigent-like protein gene in transgenic soybean hairy roots dramatically reduced plant susceptibility to cyst nematodes (Rambani et al. 2020). However, information about epigenetic control of miRNA gene expression remains elusive despite several recent studies, which have shown that altered miRNA levels can exert substantial effects on plant susceptibility to cyst and root-knot nematodes (Hewezi 2020).

Considering the key role that miRNAs play in plantnematode interactions, together with the increasing evidence of widespread DNA methylation changes in host plants in response to nematode infection, we hypothesized that SCN may induce DNA methylation changes in miRNA genes, affecting their expression and function. In this study, we analyzed DNA methylation changes of miRNA genes using SCN-resistant and -susceptible near-isogenic lines (NILs). We identified significant changes in DNA methylation levels in the promoter and primary transcript-coding regions of a significant number of miRNA genes. We show that differentially methylated miRNAs are specific to each line, affect gene expression, and modulate soybean susceptibility to SCN.

\section{RESULTS}

\section{Identification of differentially methylated miRNA genes during compatible and incompatible soybean-SCN interactions.}

To examine the extent to which miRNA genes are subjected to DNA methylation changes during the susceptible and resistant interactions of soybean with SCN, we first compared global DNA methylation levels over all annotated miRNA genes between two soybean (Glycine max) NILs that differ in their response to SCN infection (race 3). Whole-genome bisulfite sequence reads of SCN-infected and noninfected root tissues of susceptible and resistant NILs at 5 days postinfection (dpi) (Rambani et al. 2020) were mapped to the promoter and primary transcript-coding regions of all annotated soybean miRNA genes in miRBase (release 21). The promoter region was determined as $2 \mathrm{~kb}$ upstream of miRNA primary transcript (Monteys et al. 2010). Under noninfected conditions, the susceptible isogenic line (NIL-S) showed higher levels of DNA methylation over miRNA genes compared with the resistant isogenic line (NIL-R) in the CG, CHG, and CHG contexts (Supplementary Fig. S1A to C). Boxplot analysis of absolute methylation levels across miRNA genes revealed significant increases in CG and CHG methylation levels in NIL-S compared with NIL-R under noninfected conditions (Supplementary Fig. S1D).

We next compared global DNA methylation levels over miRNA genes in each line under noninfected and SCN-infected conditions. In NIL-S, a trend of global DNA hypermethylation of miRNA genes was observed in the SCN-infected root samples compared with the noninfected control, particularly in the $\mathrm{CHG}$ and $\mathrm{CHH}$ sequence contexts (Fig. 1A to C). However, in NIL-R, we detected a slight increase in global DNA methylation in the infected samples compared with noninfected samples, particularly in the CG and CHG sequence contexts (Fig. 1D to F). Furthermore, boxplot analysis of absolute methylation levels across miRNA genes in both lines upon SCN infection revealed significant increases in methylation levels in all sequence contexts in both lines, with the exception of $\mathrm{CHH}$ methylation in NIL-R (Fig. $1 \mathrm{G}$ and $\mathrm{H}$ ).

The differences in global DNA methylation of miRNA genes between NIL-S and NIL-R prompted us to identify miRNA genes that experience significant changes in DNA methylation patterns in both lines in response to SCN infection. To this end, we identified differentially methylated regions (DMRs) by dividing the miRNA promoter region into nonoverlapping windows of $500 \mathrm{bp}$ and used them to compare level and direction of DNA methylation between control and SCN-infected samples. Primary transcript regions of miRNAs were also compared between noninfected and SCN-infected samples. Regions were considered significantly differentially methylated if the methylation difference was greater than $25 \%$ with adjusted $P$ value < 0.01. In NIL-S, we identified 82 unique miRNA genes overlapping with 95 unique DMRs (Fig. 2A; Supplementary Table $\mathrm{S} 1$ ). Of these 82 miRNAs, 34 were hypermethylated and 53 were hypomethylated (Fig. 2A; Supplementary Table S1). The remaining five miRNAs overlapped with both hyper- and hypomethylated regions. In NIL-R, we identified 16 differentially methylated miRNA genes overlapping with 18 DMRs. Of these 16 miRNA, 10 were hypermethylated and 6 were hypomethylated (Fig. 2B; Supplementary Table S2). The 95 DMRs identified in NIL-S were found in the CG (28\%), CHG (48\%), and $\mathrm{CHH}(24 \%)$ sequence contexts (Fig. 2C; Supplementary Table S1). The 18 DMRs identified in NIL-R were found in the 

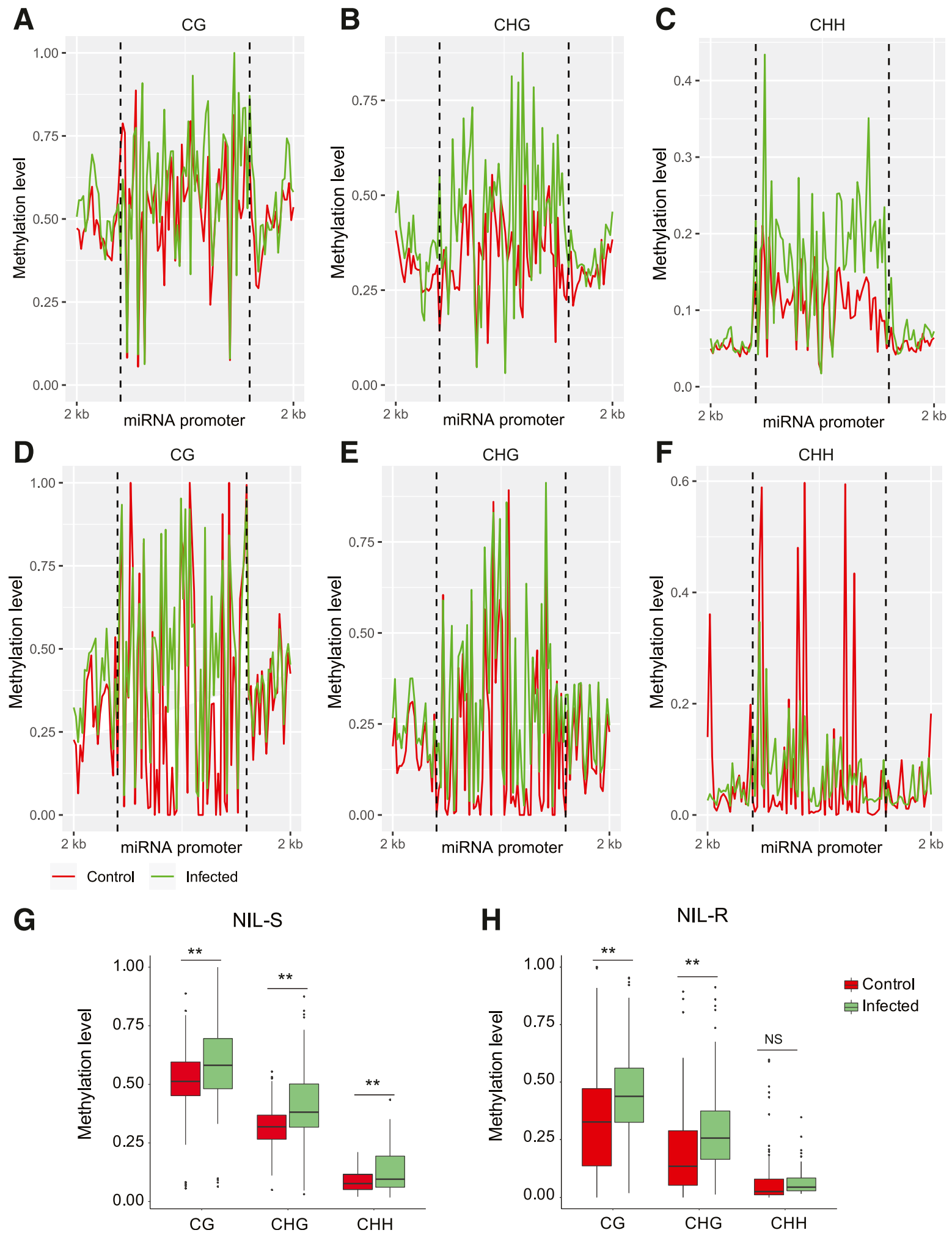

Fig. 1. Global DNA methylation levels in three sequence contexts across microRNA (miRNA) genes in the isogenic lines under soybean cyst nematode (SCN)infected and noninfected conditions. Comparison of global DNA methylation levels between SCN-infected (green) and noninfected conditions (red) in the susceptible near-isogenic line (NIL-S) over miRNA genes in the A, CG; B, CHG; and C, CHH sequence contexts. Comparison of global DNA methylation levels between SCN-infected (green) and noninfected conditions (red) in the resistant NIL (NIL-R) over miRNA genes in the D, CG; E, CHG; and F, CHH sequence contexts. Boxplots for absolute methylation levels across miRNA genes in G, NIL-S and H, NIL-R under noninfected and SCN-infected conditions in various sequence contexts. Asterisks $(* *)$ indicate $P$ value $<0.01$ with $t$ test and $\mathrm{NS}=$ not significant. Global methylation is calculated at base pair level by dividing number of methylated cytosines by total coverage. A promoter region of $2 \mathrm{~kb}$ is divided into 60 bins and methylation levels are averaged across each bin and plotted as a data point on a line graph. The y-axis represents methylation level within the range of 0 to 1 . 
CG (22\%), CHG (50\%), and CHH (28\%) contexts (Fig. 2D; Supplementary Table S2).

$\mathrm{SCN}$-induced differential methylation in miRNA genes was detected in the promoter and primary transcript-coding regions
(Fig. 2E and F). Differential DNA methylation in NIL-R occurred mainly in the promoter region, with only gma-miR5032 showing hypermethylation in the primary transcript-coding region (Fig. 2F). In NIL-S, however, 56 miRNAs showed
A

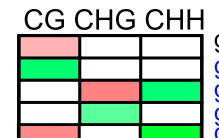

gma-miR9743

gma-miR5032

gma-miR1520b

gma-miR5043

gma-miR2107-ch3

gma-miR2107-ch14

gma-miR2107-ch2

gma-miR2107-ch5

gma-miR2107-ch6

gma-miR2107-ch13

gma-miR2107-ch13

gma-miR2107-ch18

gma-miR2107-ch19

gma-miR2107-ch

gma-miR 9722

gma-miR5377

gma-miR1518

gma-miR4371a

gma-miR 9757

gma-miR4401b

gma-miR9723

gma-miR319d
gma-miR5780c

gma-miR5780c

gma-miR4364a

gma-miR4377

gma-miR 4366
gma-miR $4371 b$

gma-miR4371b

gma-miR1520a

gma-miR390f

gma-miR 1516c

gma-miR9737

gma-miR2111b

gma-miR4345

gma-miR 9734

gma-miR4364b

gma-miR5779

gma-miR9765

gma-miR1534

gma-miR 172c

gma-miR577

gma-miR5778

gma-miR43773

gma-miR4374b

gma-miR4396

gma-miR 398

gma-miR3.

gma-miR5370

gma-miR5038

gma-miR5038b

gma-miR1521a

gma-miR1521

gma-miR $166 i$

gma-miR4380a

gma-miR 1520

gma-miR5677

gma-miR4343a

gma-miR4343a

gma-miR5770a

gma-miR395e

gma-miR4359a

gma-miR4369

gma-MIR4361
gma-miR $160 b$

gma-miR4380b

gma-miR4349

gma-miR4393a

gma-miR4399

gma-miR4387e

gma-miR 4361-ch16

gma-miR 4412

gma-miR1520f

gma-miR5670b

gma-miR 160

gma-miR4405

gma-miR1520p

gma-miR4372b

gma-miR2111f

gma-miR $1520 h$

gma-miR166h

gma-miR1513b

\section{B}

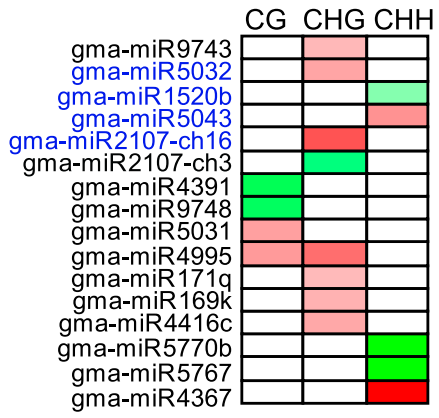

C

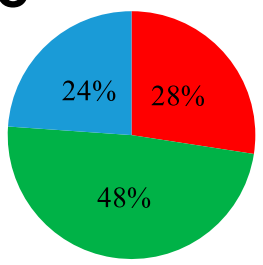

D

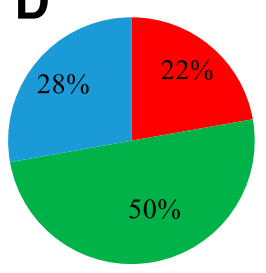

$\square \mathrm{CG}$ - $\mathrm{CHG} \square \mathrm{CHH}$

E

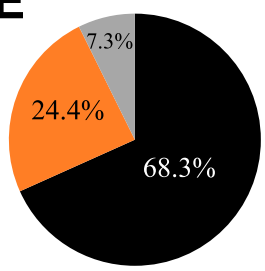

F

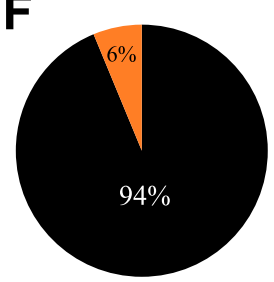

Promoter Pri-miRNA Promoter + Pri-miRNA

Fig. 2. Heatmap presentations of differentially methylated microRNAs (miRNAs) identified in the isogenic lines. A, Methylation direction and sequence contexts of 82 differentially methylated miRNAs identified in the susceptible near-isogenic line (NIL-S). B, Methylation direction and sequence contexts of 16 differentially methylated miRNAs identified in the resistant NIL (NIL-R). miRNAs showing opposite methylation patterns in the isogenic lines are highlighted in blue. Red and green in the color bar represent hypermethylation and hypomethylation, respectively. DNA methylation contexts of differentially methylated regions identified in C, NIL-S and D, NIL-R. Distribution of differentially methylated promoters and primary transcript-coding regions identified in E, NIL-S and F, NIL-R. Pri-miRNA = long primary transcript miRNA. 
differential DNA methylation in the promoter, 20 in the primary transcript-coding region, and 6 in both the promoter and primary transcript-coding regions (Fig. 2E). These results suggest that the miRNA primary transcript-coding region is less vulnerable to DNA methylation changes compared with promoter regions regardless of the size difference between these regions. We also measured the distance between the DMRs and the transcriptional start site (TSS) of miRNA genes. The average distances of the DMRs to the miRNA TSS were 556 and $647 \mathrm{bp}$ for NIL-S and NIL-R, respectively (Supplementary Tables S1 and S2).
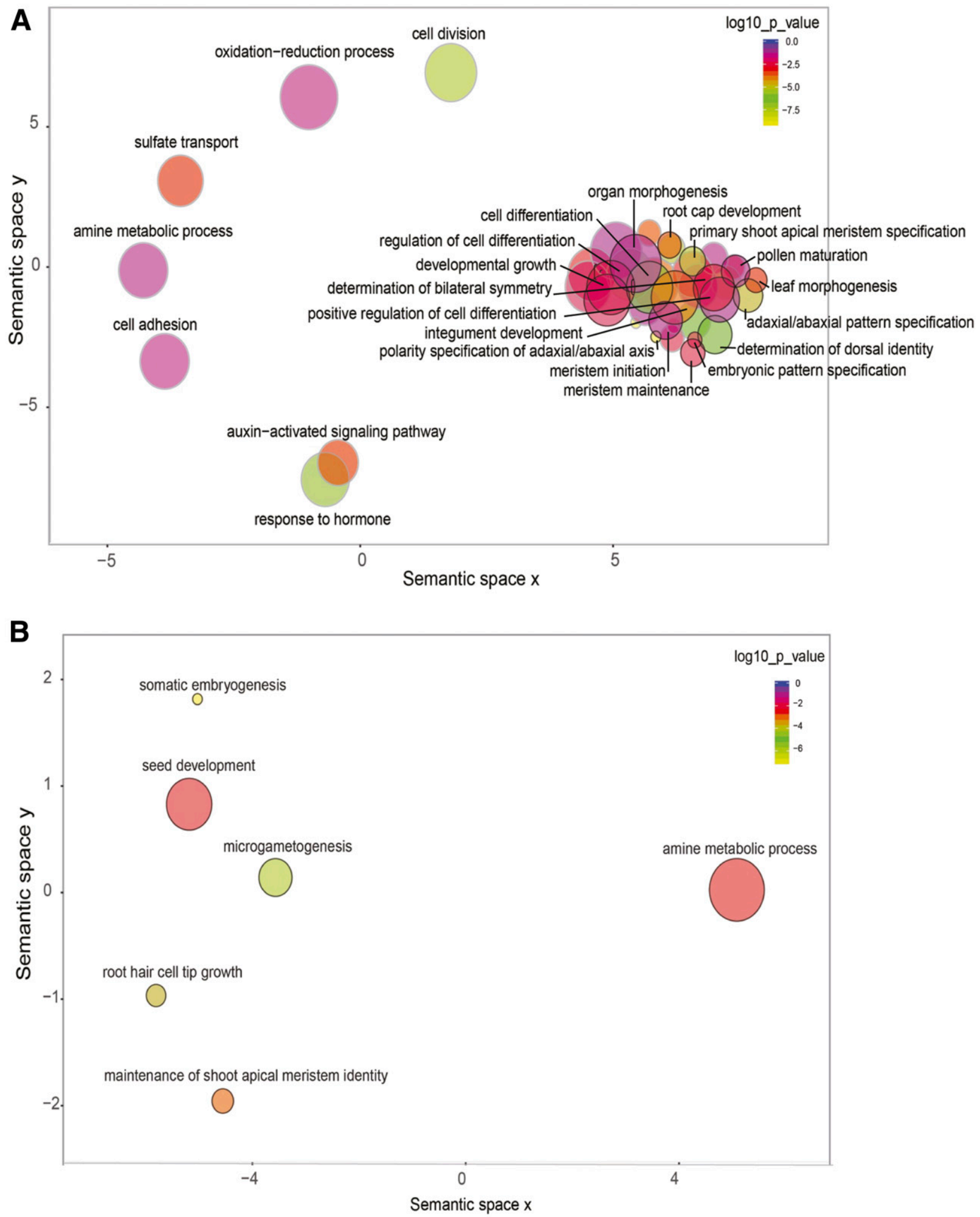

Fig. 3. Gene ontology (GO) term enrichment analysis of the target genes of differentially methylated microRNAs (miRNAs) identified in the isogenic lines. Significantly enriched biological process GO terms for target genes of differentially methylated miRNAs identified in A, the susceptible near-isogenic line (NIL-S) and B, the resistant NIL (NIL-R). GO terms are clustered based on semantic similarity to other GO terms in the Uniprot database. The size of the circle is proportional to the abundance of the GO terms in our dataset. The color bar represents $\log _{10}$ of GO term $P$ value obtained from soybase GO enrichment tool. 
TEs in miRNA gene promoters may enhance miRNA vulnerability to DNA methylation changes upon SCN infection.

We analyzed the promoter of miRNA genes for the presence of TEs to examine their potential involvement in increasing miRNA susceptibility to DNA methylation changes upon SCN infection. Interestingly, the promoters of 37 differentially methylated miRNA genes identified in NIL-S contained 92 TEs, which belong mainly to the Copia and Gypsy families of the long terminal repeat retrotransposons (Supplementary Table S3). Similarly, the promoters of five differentially methylated miRNAs identified in NIL-R contained nine TEs of the Copia and Gypsy families (Supplementary Table S4). It is noteworthy that 74 of these 99 unique TEs are among the previously identified differentially methylated TEs in these isogenic lines in response to SCN infection (Rambani et al. 2020). Therefore, the presence of TEs in the promoter of differentially methylated miRNAs may make miRNA genes prone to DNA methylation changes in response to SCN infection.

\section{Identification and functional classification of target genes of differentially methylated miRNA during compatible and incompatible interactions.}

To gain insight into the biological processes in which the differentially miRNAs function, we scanned publicly available degradome datasets to identify target genes of these miRNAs. For the differentially methylated miRNAs detected in NIL-S, we identified 165 target genes from previously published degradome datasets (Arikit et al. 2014; Ding et al. 2016; Fang et al. 2013; Hu et al. 2013; Shamimuzzaman and Vodkin 2012; Song et al. 2011; Xu et al. 2014; Xu et al. 2016; M. Zhao et al. 2015) (Supplementary Table S5). In addition a set of 167 targets were predicted using psRNATarget (Dai and Zhao 2011) (Supplementary Table S5) by applying high-stringency parameters that allow only one nucleotide mismatch between the mature miRNA sequence and its binding sites. For the differentially methylated miRNAs detected in NIL-R, 28 target genes were identified from the degradome datasets in addition to 49 predicted targets (Supplementary Table S6).

Gene ontology (GO) term enrichment analysis of the target genes of differentially methylated miRNAs identified in NIL-S revealed significant enrichment $(P$ value $<0.01)$ for 17 terms, including cell division, response to hormone stimulus, developmental growth, meristem maintenance, organ morphogenesis, sulfate transport, and regulation of cell differentiation, for instance (Fig. 3A). The identified target genes of the differentially methylated miRNAs detected in NIL-R revealed significant enrichment for five GO terms involved in amine metabolic process, maintenance of apical meristem identity, root hair cell tip growth, microgametogenesis, and somatic embryogenesis (Fig. 3B).

\section{DNA methylation affect the expression of differentially methylated miRNAs.}

When the lists of differentially-methylated miRNAs in NIL-R and NIL-S were compared, a set of five miRNAs (gmamiR9743, gma-miR1520b, gma-miR5032, gma-miR5043, and gma-miR2107-ch16) was common to both lines. gmamiR9743 was hypermethylated in both NIL-R and NIL-S in response to SCN infection. In contrast, gma-miR5032, gmamiR5043, gma-miR1520b, and gma-miR2107-ch16 had methylation change in the opposite direction in both lines (Fig. 2A and B). gma-miR5032, gma-miR5043, and gmamiR2107-ch16 were hypermethylated in NIL-R and hypomethylated in NIL-S in response to SCN infection. A DMR overlapping with the gma-miR $1520 \mathrm{~b}$ promoter was hypomethylated in NIL-R and hypermethylated in NIL-S at 5 dpi
(Fig. 2A and B; Supplementary Tables S1 and S2). In addition, the miRNA paralogs gma-miR5770a and gma-miR5770b showed opposite methylation patterns in the isogenic lines. More specifically, gma-miR5770a was hypermethylated only in NIL-S, whereas gma-miR5770b was hypomethylated only in NIL-R (Fig. 2A and B; Supplementary Tables S1 and S2). Together, the opposite methylation patterns of these four miRNAs (gma-miR1520b, gma-miR5032, gma-miR5043, and gma-miR2107-ch16) as well as gma-miR5770a and gmamiR5770b suggest a role in soybean-SCN interactions.

To examine this hypothesis, we first quantified the expression of gma-miR5032, gma-miR5043, gma-miR1520b, and gma-miR5770a/b in both lines in response to SCN infection at 3,5 , and 10 dpi using three independent biological samples of infected and noninfected root tissues. At 3 dpi, gma-miR5043 was significantly downregulated in both lines, whereas gma-miR5032, gma-miR1520b, and gma-miR5770a/b were significantly upregulated only in infected NIL-S root samples compared with noninfected samples (Fig. 4A and B). No significant changes in the expression of these miRNAs were detected in NIL-R in response to SCN at this time point (Fig. 4B). At $5 \mathrm{dpi}$, the expression patterns of the four miRNAs became very distinct in both lines. gma-miR5032 and gma-miR1520b were upregulated in NIL-S and downregulated in NIL-R (Fig. 4A and B). gma-miR5770a/b showed downregulation in both lines, whereas gma-miR5043 showed downregulation only in in NIL-R. At 10 dpi, the four miRNA genes showed slight or no significant changes in expression in both lines (Fig. 4A and B). Taken together, these data suggest that $\mathrm{SCN}$-induced differential DNA methylation in the promoter of these miRNAs at $5 \mathrm{dpi}$ would be the driving force for their gene expression changes in the isogenic lines.

Furthermore, we tested whether the miRNA gene expression changes observed upon SCN infection indirectly affect the expression of their target genes at the same time point. We quantified uncleaved transcript levels of six confirmed targets of these four miRNAs in the same RNA samples using quantitative real-time reverse-transcription PCR (qRT-PCR). These genes included Glyma.05G180700 and Glyma.08G138300 (confirmed targets of gma-miR5032), Glyma.13G270900 (confirmed target of gma-miR5043), Glyma.19G182000 (confirmed target of gma-miR1520b), and Glyma.17G019300 and Glyma.03G005400 (confirmed targets of gma-miR5770a/b) (Arikit et al. 2014; Ding et al. 2016; Fang et al. 2013; M. Zhao et al. 2015). In NIL-S, we found that significant upregulation of gma-miR5032 and gma-miR1520b at 5 dpi was associated with significant downregulation of the target genes Glyma. 05 G18070 and Glyma.19G182000, respectively (Fig. 4A and C). Similarly, a slight nonsignificant upregulation of gmamiR5043 at 5 dpi was correlated with significant downregulation of the Glyma.13G270900 target gene. Also, a significant increase in the expression of Glyma.08G138300 at 10 dpi may be related to the small downregulation of its negative regulator (gma-miR5032) at the same time point. In NIL-R, the expression of Glyma.13G270900 and Glyma.05G180700 at 3 or 5 dpi seems to be posttranscriptionally regulated by gmamiR5043 and gma-miR5032, respectively (Fig. 4B and D). Of note is that the remarkable downregulation of gma-miR5770a/b in both lines at 5 dpi was associated with upregulation of its target genes (Glyma.17G019300 and Glyma.03G005400) only in NIL-S, suggesting that additional genetic or epigenetic regulatory mechanism may be involved in the regulation of these target genes in NIL-R.

When we compared the expression of the six target genes between the isogenic lines in response to nematode infection we observed a distinct patterns of regulation. At 3 dpi, we observed significant differences in the expression of Glyma.05G180700, 
Glyma.08G138300, Glyma.13G270900, and Glyma.03G005400 between the isogenic lines in response to SCN infection (Fig. 4C and D). At 5 dpi, the expression of the six target genes was oppositely regulated in NIL-R and NIL-S (Fig. 4C and D). More specifically, whereas these target genes showed significant downregulation of approximately twofold in NIL-S, these genes showed no changes (Glyma.08G138300, Glyma.13G270900, and Glyma.19G182000) or upregulation (Glyma.05G180700, Glyma.17G019300, and Glyma.03G005400) in NIL-R (Fig. 4C and D). Similarly, at $10 \mathrm{dpi}$, differences in the expression of two target genes (Glyma.05G180700 and Glyma.08G138300) were detected between NIL-R and NIL-S (Fig. 4C and D). Together, the expression differences of these four miRNAs and their targets in NIL$\mathrm{S}$ and NIL-R, particularly at $5 \mathrm{dpi}$, are consistent with the opposite methylation patterns detected in the promoters of these miRNA genes at the same time point.

gma-miR5032, gma-miR5043, gma-miR1520b, and $\mathrm{miR5770a/b}$ are more highly expressed in NIL-R than NIL-S under noninfected conditions.

We compared the basal expression levels of gma-miR5032, gma-miR5043, gma-miR1520b, and gma-miR5770a/b in noninfected NIL-S and NIL-R control samples corresponding to 3 and $5 \mathrm{dpi}$, where differences in the expression of these miRNAs in the isogenic lines were observed upon SCN infection. Interestingly, gma-miR5032, gma-miR5043, gma-miR1520b, and gma-miR5770a/b exhibited higher expression in NIL-R relative to NIL-S at both time points, with an increase ranging between
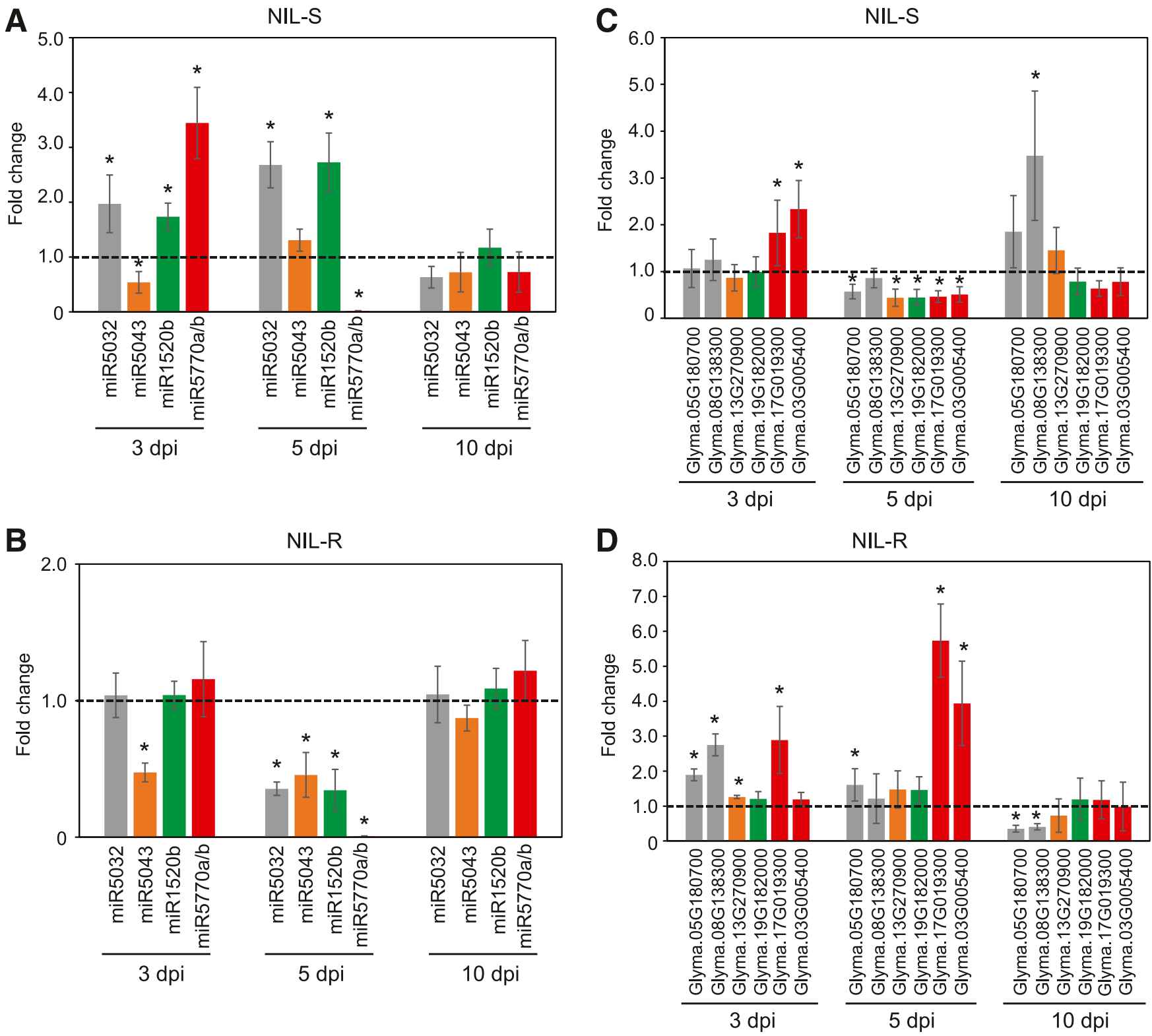

Fig. 4. Quantitative real-time PCR quantification of the expression levels of four differentially methylated microRNAs (miRNAs) and their confirmed targets in the isogenic lines in response to soybean cyst nematode (SCN) infection. Expression levels of gma-miR5032, gma-miR5043, gma-miR1520b, and gmamiR5770a/b in A, the susceptible near-isogenic line (NIL-S) and B, the resistant NIL (NIL-R) at 3, 5, and 10 days postinfection (dpi). Expression levels of six confirmed targets of gma-miR5032, gma-miR5043, gma-miR1520b, and gma-miR5770a/b in C, NIL-S and D, NIL-R at 3, 5, and 10 dpi. Expression levels of Glyma.05G180700 and Glyma.08G138300 (confirmed targets of gma-miR5032), Glyma.13G270900 (confirmed target of gma-miR5043), Glyma.19G182000 (confirmed target of gma-miR1520b), and Glyma.17G019300 and Glyma.03G005400 (confirmed targets of gma-miR5770a/b) were quantified in the same samples used in A and B. miRNA and target genes are indicated with the same color. Fold-change values represent expression levels in SCN-infected root samples relative to noninfected control samples, which were set to 1 . Shown are averages of three biologically independent samples \pm standard error. Statistically significant differences from noninfected controls were determined using $t$ tests with $P$ value $<0.05$ and indicated by an asterisk $(*)$. 
two- and 13-fold (Supplementary Fig. S2). gma-miR5043 showed approximately twofold increase in the expression in NIL-R relative to NIL-S at both time points, whereas gma-miR5032 showed more than 10-fold increase at both time points (Supplementary Fig. S2). gma-miR1520b and gma-miR5770a/b showed between 2.6- and 6.4-fold increase in the expression level in NIL-R compared with NIL-S at both time points (Supplementary Fig. S2).

\section{Functional validation}

\section{of differentially methylated miRNAs.}

We examined the potential involvement of differentially methylated miRNAs in establishing the compatibility of the interaction between soybean and SCN. To this end, the four miRNAs (gma-miR1520b, gma-miR5043, gma-miR5032, and gma-miR5770b), which showed opposite methylation patterns in the isogenic lines, were overexpressed in NIL-S using transgenic hairy root system. The primary transcript sequences of these four miRNAs were overexpressed under the control of a soybean ubiquitin promoter and the RuBisCO small subunit terminator. Transgenic hairy roots expressing the empty vector were also generated in NIL-S and used as negative controls. The transgenic hairy roots were selected with the aid of the green fluorescent protein (GFP) reporter. qPCR analysis of the mature miRNA expression in three biological samples of the transgenic hairy roots revealed at least twofold increase in the abundance of these miRNAs in the transgenic hairy roots relative to the GFP control roots (Fig. 5A). Because miRNAs function through base-paring with their target genes leading to mRNA degradation and low transcript abundance, we quantified the uncleaved transcript levels of the six aforementioned confirmed targets (Glyma.05G180700, Glyma.08G138300, Glyma.13G270900, Glyma.19G182000, Glyma.17G019300, and Glyma.03G005400) of these four miRNAs in the same transgenic hairy root samples using qPCR. The levels of uncleaved transcripts of the target genes were reduced in the miRNA overexpressed roots compared with GFP control roots (Fig. 5B), thereby validating the functionality of overexpression constructs.

We then assayed the transgenic hairy root plants to SCN susceptibility. In these experiments, each composite plant with three transgenic hairy roots was inoculated with approximately 3,000 eggs of SCN race 3 (HG type 0) and, 5 weeks after inoculation, the number of cysts per plant was counted and used to determine plant susceptibility. Overexpression of gma-miR1520b and gmamiR5043 showed approximately $30 \%$ reduction in cyst number, although this reduction is statistically insignificant (Fig. 5C). However, gma-miR5770b-overexpressing plants showed approximately $63 \%$ decrease in susceptibility to SCN. Importantly, overexpression of gma-miR5032 strongly enhanced plant resistance to SCN, with an average of 5.1 cysts per plant compared with 280.6 cysts in the susceptible control line expressing the empty vector (Fig. 5C). These results indicate that gma-miR5032 and gmamiR5770b function as positive regulators of SCN resistance.

\section{DISCUSSION}

In this study, we quantified differential DNA methylation of miRNA genes during compatible and incompatible interactions of soybean with SCN using two NILs. The use of NILs allowed us to determine the methylation differences with minimum genetic background effects. The analysis provided several insights into the differences between the resistant and susceptible responses to SCN. (i) The susceptible line is more vulnerable to DNA methylation than the resistant line because 82 miRNAs were identified as differentially methylated in NIL-S, whereas only 16 were found to be differentially methylated in NIL-R. This finding is in agreement with a previous study showing that DNA methylation changes are more dynamic in the susceptible
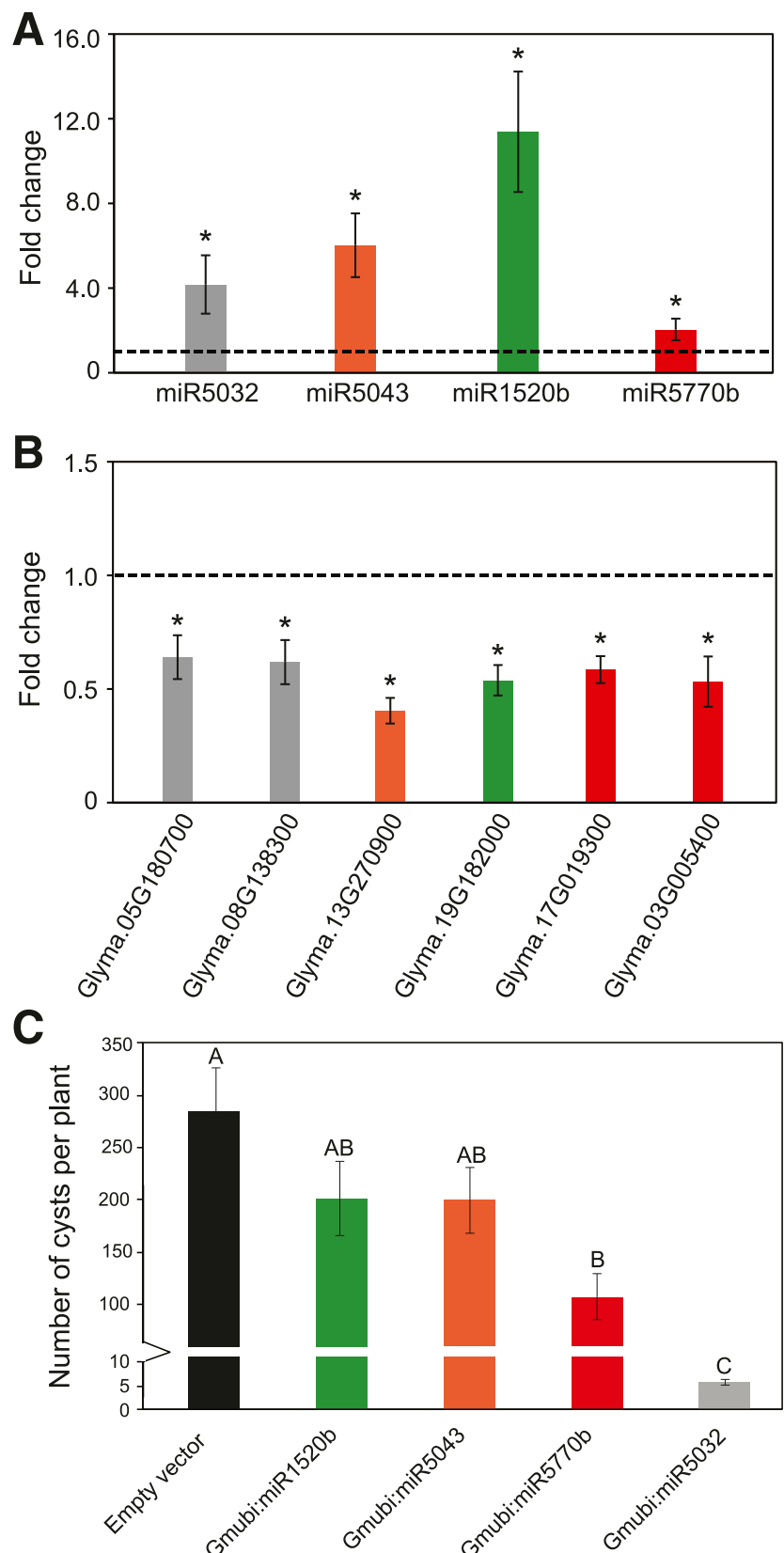

Fig. 5. Nematode susceptibility assays of microRNA (miRNA) overexpression lines. Expression levels of A, gma-miR5032, gma-miR5043, gma-miR1520b, and gma-miR5770b and B, six target genes in overexpression hairy roots samples. Fold-change values represent expression levels in the overexpression lines relative to control plants expressing the empty vector. Shown are averages of three biologically independent samples \pm standard error (SE). Statistically significant differences from control samples were determined using $t$ tests with $P$ value $<0.05$. C, Nematode susceptibility levels of miRNA overexpression hairy root lines. Transgenic hairy roots overexpressing gma-miR5032, gma-miR5043, gma-miR1520b, and gma-miR5770b were generated in the susceptible near-isogenic line (NIL-S). Each composite plant was inoculated with approximately 3,000 eggs of soybean cyst nematode $(\mathrm{SCN})$ race 3 per plant and, 5 weeks postinoculation, the number of cysts per plant were counted. Composite NIL-S plants expressing the empty vector were used as susceptible control. Data are averages of three replicates, each with at least three plants, \pm SE. Statistically significant differences between miRNA overexpressing plants and the control were determined using analysis of variance. Bars with different letters are statistically significantly different $(P<0.05)$ from control plants expressing the empty vector. 
interaction than the resistant interaction (Rambani et al. 2020). (ii) DMRs overlapping with miRNA genes showed opposite trends of regulation during resistant and susceptible interactions (Fig. 2) despite both lines showed increased global DNA methylation over miRNA genes in response to SCN infection (Fig. 1). Whereas, in NIL-S, hypo-DMRs overlapping with the miRNA gene promoter are more prevalent than hypermethylation, the opposite trend was observed in NIL-R (Fig. 2). (iii) Differential DNA methylation occurs more predominantly in miRNA promoter than primary transcript-coding regions. This is the opposite of protein-coding genes, where methylation changes in gene body regions occur more predominantly than gene promoters during plant interactions with cyst nematodes (Hewezi et al. 2017; Rambani et al. 2015, 2020). Although DNA methylation in gene promoters is known to contribute to the control of gene transcription by affecting transcription factor accessibility, the biological significance of DNA methylation of miRNA primary transcript-coding regions remains largely unknown. However, DNA methylation of miRNA primary transcript-coding regions may have a role in miRNA biogenesis, as recently reported in mammalian cells (Glaich et al. 2019). In this scenario, DNA methylation in the primary miRNA transcript-coding region may slow the elongation rate of PolII, thereby providing the necessary time for newly synthesized primary miRNA transcripts to be processed into mature molecules (Glaich et al. 2019). (iv) Twothirds of DMRs overlapping with miRNA genes were found in non-CG sequence contexts, consistent with the predominance of CHG and $\mathrm{CHH}$ methylation in gene promoters (Lindroth et al. 2001). (v) Methylation changes in miRNA genes were unique between the isogenic lines. Only five miRNA genes were found to be differentially methylated in both lines. However, four (gmamiR5032, gma-miR5043, gma-miR1520b, and gma-miR2107ch16) of these five miRNAs showed opposite methylation patterns in the isogenic lines.

One obvious question raised from our analysis is whether DNA methylation levels across miRNAs are equivalent to those observed for protein-coding genes and TEs. Under noninfected conditions, the global methylation levels over miRNAs were higher in NIL-S compared with NIL-R (Supplementary Fig. S1) and follow the same patterns previously reported in the isogenic lines for protein-coding genes and TEs, particularly in non-CG contexts (Rambani et al. 2020). Under SCN-infected conditions, NIL-R exhibited increased global methylation levels across miRNA genes, similar to what was previously reported for protein-coding genes and TEs in NIL-R upon SCN infection (Rambani et al. 2020). SCN-infected NIL-S also exhibited increased global methylation levels over miRNAs, even though the same line showed global hypomethylation over protein-coding genes and TEs upon SCN infection (Rambani et al. 2020). Despite our finding that global methylation levels were increased over miRNAs in SCN-infected NIL-S, the majority of DMRs were hypomethylated. Similarly, hypo-DMRs overlapping with genes and TEs in NIL-S were more prevalent than hyper-DMRs in response to SCN infection (Rambani et al. 2020).

Compared with Arabidopsis, the genomes of crop plants such as soybean contain higher content of TEs, many of which are located close to genes, making these genes more vulnerable to DNA methylation changes (Zhang et al. 2018). The presence of 99 TEs in the promoter of 41 differentially methylated miRNA genes may have contributed to the increased level of DNA methylation over miRNAs and their vulnerability to DNA methylation changes upon SCN infection. This suggestion is consistent with our finding that 74 of these 99 TEs have been previously identified as differentially methylated in these isogenic lines upon SCN infection (Rambani et al. 2020). In addition, our finding that the large majority of the TEs located in the promoter of differentially methylated miRNA genes belongs to the Copia and Gypsy families is in line with our recent findings that retrotransposons tend to be more vulnerable to DNA methylation change upon cyst nematode infection than other types of TEs (Hewezi et al. 2017; Piya et al. 2017a; Rambani et al. 2020).

Positive and negative associations between DNA methylation of TEs and the expression of nearby genes were reported in several studies (Dowen et al. 2012; Hewezi et al. 2017; Le et al. 2014; Wang et al. 2013). DNA methylation of TEs located in miRNA gene promoters may affect the expression of miRNA genes by inhibiting TE-generated cryptic transcripts, which may interfere with miRNA transcription. TE methylation may also alter chromatin structure of miRNA gene promoters, directly affecting miRNA transcription. In addition, in some cases, TEs can function as cis-regulatory elements (Makarevitch et al. 2015), and their methylation status may alter the binding ability of transcriptional activators or repressors, thereby affecting miRNA transcription.

The expression levels of gma-miR5032 and gma-miR5043 in the isogenic lines are consistent with the anticipated negative association between DNA methylation and gene expression (Zhang et al. 2018). However, we detected a positive association between DNA methylation and gene expression for gmamiR1520b. This finding may be related the characteristics of the DMRs, including methylation level and sequence context, as well as the presence of regulatory elements of negative regulators and repressive chromatin marks where hypermethylation may result in gene upregulation and hypomethylation may result in gene downregulation (Gent et al. 2013; Hewezi et al. 2017; Rambani et al. 2015; Zhang et al. 2018). Another possibility is that upregulation of gmamiR1520b in NIL-S at 5 dpi could be due to recognition of the hypermethylated $\mathrm{CHG}$ region in the promoter by specific transcription factors that possess specific binding activity to methylated DNA-binding elements, as reported for human transcription factors (Yin et al. 2017). The association between DNA methylation and the expression of gma-miR5770a/b in the isogenic lines can be explained by the possibility that partial hypomethylation of gma-miR5770b in a 500-bp region in response to SCN was not sufficient for gma-miR5770b upregulation in NIL-R. In addition, increased hypermethylation of gma-miR5770a in NIL-S in response to SCN infection only increased the level of gma-miR5770a downregulation. Though our data point to a possible role of DNA methylation in regulating the expression of these miRNAs and, indirectly, their targets, we don't rule out the possibility that other genetic or epigenetic regulatory mechanisms may also play a role.

Several lines of evidence implicate the identified differentially methylated miRNAs between the isogenic lines upon SCN infection in plant-SCN interactions. Many of the identified differentially methylated miRNAs have been shown to change their abundance in response to SCN infection (Lei et al. 2019; Li et al. 2012; Tian et al. 2017). In addition, several of miRNA targeted genes were previously reported as SCN responsive and include genes encoding functions important for nematode infection. It is remarkable that the identified target genes are enriched in functions related to cell differentiation, organ morphogenesis, and cell fate and specification (Fig. 3A). Because these functions are vital for syncytium formation and development (Hewezi 2015), it is tempting to speculate on the importance of these differentially methylated miRNAs in establishing nematode feeding sites and, hence, the compatibility of soybean-SCN interactions. Furthermore, we noted that a significant number of the miRNA target genes are transcription factors belonging to various families. This includes auxin response, MYB, bHLH, GRAS, scarecrow, NAC, and nuclear factor $\mathrm{Y}$ family transcription factors, for instance (Supplementary Tables S5 and S6). This observation holds true for both 
isogenic lines, and suggests a role of differentially methylated miRNAs in transcriptome reprogramming during nematode infection of soybean plants. Previous reports have established miRNAs as key mediators of global transcriptome change during nematode infection by targeting plant transcription factors (Hewezi et al. 2008, 2012; Li et al. 2012; Medina et al. 2017; Piya et al. 2017b). Among the differentially methylated miRNAs, we found miR393 (Wong et al. 2014), miR172 (Q. Zhao et al. 2015), miR319 (Zhang et al. 2016; W. Zhao et al. 2015), and miR160 (Natarajan et al. 2018), whose roles in modulating plant defense response are well established.

Our functional assays provided evidence for the involvement of differentially methylated miRNAs in soybean-SCN interactions. Overexpression of gma-miR5770b and gma-miR5032 significantly enhanced plant resistance. In this context, it may be relevant to mention that the basal expression levels of these miRNAs were much higher in the NIL-R compared with NIL-S under noninfected conditions. Thus, it is reasonable to expect that constitutive overexpression of gma-miR5032 and gmamiR5770b in NIL-S would surpass the slight increases in their expression observed in this line at 3 or $5 \mathrm{dpi}$, leading to plant resistance. gma-miR5770b targets two copper amine oxidase family proteins (Glyma.17 G019300 and Glyma.02G120600) (Ding et al. 2016; Fang et al. 2013), among other genes. Copper amine oxidase ( $\mathrm{CuAO}$; EC 1.4.3.6) catalyzes the oxidative deamination of polyamines. It has been shown that cyst nematodes modulate polyamine metabolism and signaling via the $10 \mathrm{~A} 06$ effector protein (Hewezi et al. 2010), and the potential involvement of gma-miR5770b in modulating polyamine concentration suggests a role of DNA methylation of gma-miR5770b in this modulation. Oxidative deamination of biologically active amines by copper amine oxidases results in the production of hydrogen peroxide, a signaling molecule known to be involved in various aspects of plant growth and defense responses (Neill et al. 2002; Smirnoff and Arnaud 2019). The generated hydrogen peroxide at a low concentration consequent to amine degradation may enhance antioxidant machinery in the syncytial cells, leading to successful nematode parasitism (Hewezi et al. 2010; Siddique et al. 2014). Thus, it is reasonable to speculate that downregulation of copper amine oxidase genes in transgenic hairy roots overexpressing gma-miR5770b eliminates this protective function, leading to syncytium degradation and plant resistance. It may be important to mention that previous studies have also linked amine oxidases to various aspects of plant defenses, including cell death, cell wall lignification, and oxidative burst (Cona et al. 2006). However, the reduced nematode susceptibility of gma-miR5770b-overexpressing plants where Glyma.17G019300 and Glyma.03G005400 were downregulated argues against a role of these copper amine oxidases in positively regulating defense responses.

The effect of gma-miR5032 overexpression on providing robust resistance against SCN is remarkable. Two genes (Glyma.05G180700 and Glyma.08G138300) encoding glycosyl hydrolases have been confirmed as targets of gma-miR5032 (M. Zhao et al. 2015). This enzyme is involved in the breakdown of sucrose and other complex carbohydrate molecules into smaller molecules such as glucose, which is considered the major source of carbohydrate input in the syncytium (Cabello et al. 2014; Hofmann et al. 2009). Therefore, one can speculate that constitutive downregulation of these enzymes in gmamiR5032-overexpressing plants may affect sugar pools in the syncytium, leading to nematode starvation and plant resistance.

In conclusion, our study demonstrates that SCN can induce hyper-and hypomethylation in miRNA genes, particularly those with functions related to plant response to nematode infection. DNA methylation can regulate miRNA expression in a manner that provides cell-type-specific and infection-stage-specific expression. Another possibility is that DNA methylation may regulate the steady-state expression of tightly regulated miRNAs, preventing aberrant induction or repression during the infection. Therefore, modulating and fine tuning the expression levels of miRNA genes in the nematode feeding sites through DNA methylation may facilitate or hinder nematode infection. Because several miRNAs have been established as major regulators of plant-nematode interactions, further studies are needed to better understand the underlying mechanisms of epigenetic regulation of miRNA expression and how interference with this regulation may enhance plant resistance.

\section{MATERIALS AND METHODS}

\section{Plant materials.}

Two soybean NILs (TN09-16 and TN09-29) were previously described (Rambani et al. 2020). TN09-16 is susceptible to SCN (race 3), whereas TN09-29 is resistant. As early as 5 dpi, the differences between the NILs were observed because NIL$\mathrm{R}$ showed a Peking-type resistance of strong syncytium degradation (Rambani et al. 2020).

\section{Differential DNA methylation analysis of miRNA genes.}

Trimmed and high-quality bisulfite sequencing reads of the near isogenic lines under SCN infected and noninfected conditions (Rambani et al. 2020) were mapped to Glycine max assembly version 1 (JGI Phytozome database) using Bismark (Krueger and Andrews 2011). Annotation of miRNA genes in soybean genome was downloaded from miRBase (release 21) to obtain coordinates of the primary miRNA gene body in the soybean genome assembly. The promoter of miRNA was defined $2 \mathrm{~kb}$ upstream of primary miRNA start site. The R package methylKit (Akalin et al. 2012) was used to identify DMRs in miRNA promoter and primary transcript-coding regions. The 2$\mathrm{kb}$ promoter region was further split into four bins of $500 \mathrm{bp}$ for accurate detection of differential methylation. Regions were considered significantly differentially methylated if the methylation difference was greater than $25 \%$ with adjusted $P$ value $<$

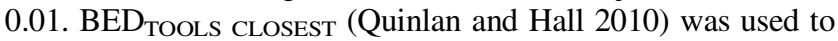
identify TEs that overlap with the promoters of differentially methylated miRNAs and calculate their distance from the miRNA transcriptional start site. Metaplots representing global DNA levels over miRNA genes in various sequence contexts were generated using the ViewBS package (Huang et al. 2018) and the MethOverRegion function. Absolute methylation data extracted from ViewBS using the MethOverRegion function were used to generate boxplots representing global DNA methylation levels over miRNA genes using the $\mathrm{R}$ package ggplot2.

\section{Identification of miRNA targets and their functional analysis.}

The psRNA Target server (Dai and Zhao 2011) was used to identify targets of differentially methylated miRNAs. Mature miRNA sequences of differentially methylated miRNAs were used to search for complementarity sequences in all annotated soybean protein-coding genes. The alignment was performed with default parameters allowing only one mismatch in the binding region. An alignment score was calculated considering miRNA length, penalizing for G:U pairing, and opening and extending a gap. Genes with an alignment score of less than 3.0 were considered as potential miRNA targets. Targets of differential miRNAs were also identified from the sequencing degradome dataset. We compiled data from various publicly available soybean degradome datasets (Arikit et al. 2014; Ding et al. 2016; Fang et al. 2013; Hu et al. 2013; Shamimuzzaman and Vodkin 2012; Song 
et al. 2011; Xu et al. 2014; Xu et al. 2016; M. Zhao et al. 2015) and searched for targets of the differentially methylated miRNAs. GO of target genes was determined using the SoyBase toolshed and was visualized using REVIGO (Supek et al. 2011).

\section{Construction of miRNA overexpression vectors.}

The primary transcripts of gma-miR1520b, gma-miR5043, gma-miR5032, and gma-miR5770b were amplified from genomic DNA using a primer pair containing the BamHI and AscI restriction sites (Supplementary Table S7). The PCR conditions used were $94^{\circ} \mathrm{C}$ for $2 \mathrm{~min}$; followed by 35 cycles at $94^{\circ} \mathrm{C}$ for 30 $\mathrm{s}, 57^{\circ} \mathrm{C}$ for $30 \mathrm{~s}$, and $72^{\circ} \mathrm{C}$ for $1 \mathrm{~min}$; and a final extension at $72^{\circ} \mathrm{C}$ for $10 \mathrm{~min}$. The PCR products were digested, purified, and ligated into the binary vector pG2RNAi2 under control of the Gmubi promoter. The pG2RNAi2 vector contains the GFP as a selectable marker. All sequences were confirmed by sequencing. The binary vectors containing the miRNA primary transcripts as well as the empty pG2RNAi2 vector were introduced into Agrobacterium rhizogenes strain K599 for generating transgenic hairy roots.

\section{Generation of transgenic soybean hairy roots.}

Soybean seed of NIL-S were surface sterilized with bleach and placed evenly spaced on a sheet of wet germination paper. Healthy germinated seedlings were transferred to an 18-cell germination tray containing sterile autoclaved vermiculite. Five-day-old seedlings were injected with strain K599 of A. rhizogenes containing the binary vectors. A bacterial cell suspension in distilled water was injected into the hypocotyl of soybean seedlings using a $3-\mathrm{ml}$ syringe needle. The inoculated seedlings were grown in a growth chamber at $26^{\circ} \mathrm{C}$ with a cycle of $16 \mathrm{~h}$ of light and $8 \mathrm{~h}$ of darkness. Approximately 4 to 5 weeks after inoculation, the transgenic hairy roots were selected using GFP with the aid of an epifluorescent microscope (Olympus stereomicroscope, model SZX12; Olympus America, Center Valley, PA, U.S.A.). Soybean plants with at least three transgenic hairy roots were used for SCN susceptibility assays. Transgenic roots were also collected in three biological replicates for RNA isolation and qPCR quantification of the expression levels of miRNAs and their target genes.

\section{SCN susceptibility assay.}

The nematode susceptibility assays were conducted as described by Rambani et al. (2020). In brief, the transgenic hairy roots overexpressing gma-miR1520b, gma-miR5043, gmamiR5032, and gma-miR5770b as well as the empty vector were planted in $656-\mathrm{cm}^{3}$ cone-tainers $(6.4$ by $25.4 \mathrm{~cm})$ filled with steam-sterilized sand and top soil at a 3:1 ratio. The plants were arranged in a randomized complete block design with three replicates, each with at least three plants. Each plant was inoculated with approximately 3,000 eggs of SCN race 3 (HG type 0 ). Approximately 5 weeks postinoculation, the cysts were extracted from each plant separately and used to determine susceptibility level in miRNA overexpression plants relative to negative control plants expressing the empty vector. Statistically significant differences between miRNA overexpression plants and control were determined using analysis of variance.

\section{Nematode inoculation, RNA isolation, and qPCR quantifications.}

Soybean seed were surface sterilized and then germinated on germination paper at $26^{\circ} \mathrm{C}$ in darkness. After 3 days, healthy seedlings were inoculated with approximately 3,000 secondstage juvenile of SCN HG type 0 (race 3) per seedling. The plants were transferred to a plant growth chamber at $26^{\circ} \mathrm{C}$ with $16 \mathrm{~h}$ of light and $8 \mathrm{~h}$ of darkness. Three biological samples of root tissues were collected at 3,5 , and 10 days after nematode inoculation from SCN-infected plants and the corresponding noninfected controls. The tissues were flash frozen, ground to fine powder, and then used for RNA isolation.

To quantify the mature miRNA, total RNA was isolated from $500 \mathrm{mg}$ root tissues using TRIzol reagent (Invitrogen) following the manufacturer's instructions. RNA was reversely transcribed into cDNA using Mir-X miRNA First-Strand Synthesis Kit (Clontech) according to the manufacturer's instructions. For each assay of qRT-PCR, approximately $25 \mathrm{ng}$ of synthesized cDNA was used as a template. qRT-PCR assays were performed in QuantStudio 6 Flex Real-Time PCR System (Applied Biosystems) using TB Green Advantage qPCR Premix (Clontech) following the manufacturer's instructions. The PCR was performed as follows: $95^{\circ} \mathrm{C}$ for $3 \mathrm{~min}$ and 40 cycles of $95^{\circ} \mathrm{C}$ for $30 \mathrm{~s}$ and $60^{\circ} \mathrm{C}$ for $30 \mathrm{~s}$. Dissociation curves were generated to determine amplification specificity. The dissociation program was $95^{\circ} \mathrm{C}$ for $15 \mathrm{~s}$ and $50^{\circ} \mathrm{C}$ for $15 \mathrm{~s}$, followed by a slow gradient from 50 to $95^{\circ} \mathrm{C}$.

To quantify the targets of miRNAs, total RNA was isolated from $500 \mathrm{mg}$ of root tissue according to Verwoerd et al. (1989). Total RNA was treated with DNase I (Invitrogen). RT-PCR assays were performed in the QuantStudio 6 Flex Real-Time PCR System (Applied Biosystems) using the Verso 1-step RT-qPCR Low ROX Kit (Thermo Scientific) according to the manufacturer's protocol. The PCR program was $50^{\circ} \mathrm{C}$ for $15 \mathrm{~min}$, followed by $95^{\circ} \mathrm{C}$ for $15 \mathrm{~min}$ and then 40 cycles of $95^{\circ} \mathrm{C}$ for $15 \mathrm{~s}, 60^{\circ} \mathrm{C}$ for $90 \mathrm{~s}$, and $72^{\circ} \mathrm{C}$ for $30 \mathrm{~s}$. The dissociation curves were generated using the following program: $95^{\circ} \mathrm{C}$ for $15 \mathrm{~s}$ and $60^{\circ} \mathrm{C}$ for $75 \mathrm{~s}$, followed by a slow gradient from 60 to $95^{\circ} \mathrm{C}$. Small nuclear RNA U6 and $60 \mathrm{~S}$ were used to normalize the expression levels of miRNAs, whereas soybean ubiquitin (Glyma.20G141600) and $\beta$-actin (Glyma. $15 \mathrm{G} 050200$ ) were used to normalize the expression levels of target genes (Rambani et al. 2015; Wan et al. 2015). Gene expression levels were analyzed using the $2^{-\Delta \Delta C T}$ method (Livak and Schmittgen 2001). Primer sequences used in qPCR assays are provided in Supplementary Table S7.

\section{Data availability.}

MethylC-Seq data are available the National Center for Biotechnology Information database under accession number GSE130134.

\section{AUTHOR-RECOMMENDED INTERNET RESOURCES}

miRBase database: http://www.mirbase.org

JGI Phytozome database: https://phytozome.jgi.doe.gov/pz/portal.html The psRNA Target server: http://plantgrn.noble.org/psRNATarget

SoyBase toolshed: https://soybase.org/goslimgraphic_v2/dashboard.php

\section{LITERATURE CITED}

Akalin, A., Kormaksson, M., Li, S., Garrett-Bakelman, F. E., Figueroa, M. E., Melnick, A., and Mason, C. E. 2012. methylKit: A comprehensive $\mathrm{R}$ package for the analysis of genome-wide DNA methylation profiles. Genome Biol. 13:R87.

Arikit, S., Xia, R., Kakrana, A., Huang, K., Zhai, J., Yan, Z., Valdés-López, O., Prince, S., Musket, T. A., Nguyen, H. T., Stacey, G., and Meyers, B. C. 2014. An atlas of soybean small RNAs identifies phased siRNAs from hundreds of coding genes. Plant Cell 26:4584-4601.

Atighi, M. R., Verstraeten, B., De Meyer, T., and Kyndt, T. 2020. Genomewide DNA hypomethylation shapes nematode pattern-triggered immunity in plants. New Phytol. 227:545-558.

Attwood, J. T., Yung, R. L., and Richardson, B. C. 2002. DNA methylation and the regulation of gene transcription. Cell. Mol. Life Sci. 59:241-257.

Bartel, D. P. 2004. MicroRNAs: Genomics, biogenesis, mechanism, and function. Cell 116:281-297.

Boyko, A., Blevins, T., Yao, Y., Golubov, A., Bilichak, A., Ilnytskyy, Y., Hollunder, J., Meins, F., Jr., and Kovalchuk, I. 2010. Transgenerational adaptation of Arabidopsis to stress requires DNA methylation and the function of Dicer-like proteins. PLoS One 5:e9514. 
Cabello, S., Lorenz, C., Crespo, S., Cabrera, J., Ludwig, R., Escobar, C., and Hofmann, J. 2014. Altered sucrose synthase and invertase expression affects the local and systemic sugar metabolism of nematode-infected Arabidopsis thaliana plants. J. Exp. Bot. 65:201-212.

Choi, J., Lyons, D. B., Kim, M. Y., Moore, J. D., and Zilberman, D. 2020 DNA methylation and histone $\mathrm{H} 1$ jointly repress transposable elements and aberrant intragenic transcripts. Mol. Cell 77:310-323.e7.

Ci, D., Song, Y., Tian, M., and Zhang, D. 2015. Methylation of miRNA genes in the response to temperature stress in Populus simonii. Front. Plant Sci. 6:921.

Cona, A., Rea, G., Angelini, R., Federico, R., and Tavladoraki, P. 2006. Functions of amine oxidases in plant development and defence. Trends Plant Sci. 11:80-88.

Dai, X., and Zhao, P. X. 2011. psRNATarget: A plant small RNA target analysis server. Nucleic Acids Res. 39:W155-W159.

Ding, X., Li, J., Zhang, H., He, T., Han, S., Li, Y., Yang, S., and Gai, J. 2016. Identification of miRNAs and their targets by high-throughput sequencing and degradome analysis in cytoplasmic male-sterile line NJCMS1A and its maintainer NJCMS1B of soybean. BMC Genomics 17:24.

Dowen, R. H., Pelizzola, M., Schmitz, R. J., Lister, R., Dowen, J. M., Nery, J. R., Dixon, J. E., and Ecker, J. R. 2012. Widespread dynamic DNA methylation in response to biotic stress. Proc. Natl. Acad. Sci. U.S.A. 109:E2183-E2191.

Fang, X., Zhao, Y., Ma, Q., Huang, Y., Wang, P., Zhang, J., Nian, H., and Yang, C. 2013. Identification and comparative analysis of cadmium tolerance-associated miRNAs and their targets in two soybean genotypes. PLoS One 8:e81471.

Feng, S., Cokus, S. J., Zhang, X., Chen, P.-Y., Bostick, M., Goll, M. G., Hetzel, J., Jain, J., Strauss, S. H., Halpern, M. E., Ukomadu, C., Sadler, K. C., Pradhan, S., Pellegrini, M., and Jacobsen, S. E. 2010. Conservation and divergence of methylation patterning in plants and animals. Proc. Natl. Acad. Sci. U.S.A. 107:8689-8694.

Gent, J. I., Ellis, N. A., Guo, L., Harkess, A. E., Yao, Y., Zhang, X., and Dawe, R. K. 2013. CHH islands: De novo DNA methylation in near-gene chromatin regulation in maize. Genome Res. 23:628-637.

Glaich, O., Parikh, S., Bell, R. E., Mekahel, K., Donyo, M., Leader, Y., Shayevitch, R., Sheinboim, D., Yannai, S., Hollander, D., Melamed, Z., Lev-Maor, G., Ast, G., and Levy, C. 2019. DNA methylation directs microRNA biogenesis in mammalian cells. Nat. Commun. 10:5657.

Han, L., Witmer, P. D. W., Casey, E., Valle, D., and Sukumar, S. 2007. DNA methylation regulates microRNA expression. Cancer Biol. Ther. 6: 1290-1294.

He, X. J., Chen, T., and Zhu, J. K. 2011. Regulation and function of DNA methylation in plants and animals. Cell Res. 21:442-465.

Hewezi, T. 2015. Cellular signaling pathways and posttranslational modifications mediated by nematode effector proteins. Plant Physiol. 169:1018-1026.

Hewezi, T. 2020. Epigenetic mechanisms in nematode-plant interactions. Annu. Rev. Phytopathol. 58:119-138.

Hewezi, T., Howe, P., Maier, T. R., and Baum, T. J. 2008. Arabidopsis small RNAs and their targets during cyst nematode parasitism. Mol. PlantMicrobe Interact. 21:1622-1634.

Hewezi, T., Howe, P. J., Maier, T. R., Hussey, R. S., Mitchum, M. G., Davis, E. L., and Baum, T. J. 2010. Arabidopsis spermidine synthase is targeted by an effector protein of the cyst nematode Heterodera schachtii. Plant Physiol. 152:968-984.

Hewezi, T., Lane, T., Piya, S., Rambani, A., Rice, J. H., and Staton, M. 2017. Cyst nematode parasitism induces dynamic changes in the root epigenome. Plant Physiol. 174:405-420.

Hewezi, T., Maier, T. R., Nettleton, D., and Baum, T. J. 2012. The Arabidopsis microRNA396-GRF1/GRF3 regulatory module acts as a developmental regulator in the reprogramming of root cells during cyst nematode infection. Plant Physiol. 159:321-335.

Hewezi, T., Pantalone, V., Bennett, M., Neal Stewart, C., Jr., and BurchSmith, T. M. 2018. Phytopathogen-induced changes to plant methylomes. Plant Cell Rep. 37:17-23.

Hofmann, J., Hess, P. H., Szakasits, D., Blöchl, A., Wieczorek, K., Daxböck-Horvath, S., Bohlmann, H., van Bel, A. J. E., and Grundler, F. M. W. 2009. Diversity and activity of sugar transporters in nematodeinduced root syncytia. J. Exp. Bot. 60:3085-3095.

Hu, Z., Jiang, Q., Ni, Z., Chen, R., Xu, S., and Zhang, H. 2013. Analyses of a Glycine max degradome library identify microRNA targets and microRNAs that trigger secondary siRNA biogenesis. J. Integr. Plant Biol. 55:160-176

Huang, X., Zhang, S., Li, K., Thimmapuram, J., Xie, S., and Wren, J. 2018. ViewBS: A powerful toolkit for visualization of high-throughput bisulfite sequencing data. Bioinformatics 34:708-709.
Ju, Z., Jiang, Q., Wang, J., Wang, X., Yang, C., Sun, Y., Zhang, Y., Wang, C., Gao, Y., Wei, X., Hou, M., and Huang, J. 2020. Genome-wide methylation and transcriptome of blood neutrophils reveal the roles of DNA methylation in affecting transcription of protein-coding genes and miRNAs in E. coli-infected mastitis cows. BMC Genomics 21:102.

Krueger, F., and Andrews, S. R. 2011. Bismark: A flexible aligner and methylation caller for Bisulfite-Seq applications. Bioinformatics 27: 1571-1572.

Le, T. N., Schumann, U., Smith, N. A., Tiwari, S., Au, P. C. K., Zhu, Q. H., Taylor, J. M., Kazan, K., Llewellyn, D. J., Zhang, R., Dennis, E. S., and Wang, M. B. 2014. DNA demethylases target promoter transposable elements to positively regulate stress responsive genes in Arabidopsis. Genome Biol. 15:458.

Lei, P., Han, B., Wang, Y. Y., Zhu, X. F., Xuan, Y. H., Liu, X. Y., Fan, H. Y., Chen, L. J., and Duan, Y. X. 2019. Identification of microRNAs that respond to soybean cyst nematode infection in early stages in resistant and susceptible soybean cultivars. Int. J. Mol. Sci. 20:5634.

Li, X., Wang, X., Zhang, S., Liu, D., Duan, Y., and Dong, W. 2012. Identification of soybean microRNAs involved in soybean cyst nematode infection by deep sequencing. PLoS One 7:e39650.

Lindroth, A. M., Cao, X., Jackson, J. P., Zilberman, D., McCallum, C. M., Henikoff, S., and Jacobsen, S. E. 2001. Requirement of CHROMOMETHYLASE3 for maintenance of CpXpG methylation. Science 292:2077-2080.

Livak, K. J., and Schmittgen, T. D. 2001. Analysis of relative gene expression data using real-time quantitative PCR and the $2^{-\Delta \Delta C T}$ method. Methods 25:402-408

Makarevitch, I., Waters, A. J., West, P. T., Stitzer, M., Hirsch, C. N., Ross-Ibarra, J., and Springer, N. M. 2015. Transposable elements contribute to activation of maize genes in response to abiotic stress. PLoS Genet. 11:e1004915.

Medina, C., da Rocha, M., Magliano, M., Ratpopoulo, A., Revel, B. Marteu, N., Magnone, V., Lebrigand, K., Cabrera, J., Barcala, M., Silva, A. C., Millar, A., Escobar, C., Abad, P., Favery, B., and JaubertPossamai, S. 2017. Characterization of microRNAs from Arabidopsis galls highlights a role for miR159 in the plant response to the root-knot nematode Meloidogyne incognita. New Phytol. 216:882-896.

Mo, Z., Li, Q., Cai, L., Zhan, M., and Xu, Q. 2019. The effect of DNA methylation on the miRNA expression pattern in lipopolysaccharideinduced inflammatory responses in human dental pulp cells. Mol. Immunol. 111:11-18.

Monteys, A. M., Spengler, R. M., Wan, J., Tecedor, L., Lennox, K. A., Xing, Y., and Davidson, B. L. 2010. Structure and activity of putative intronic miRNA promoters. RNA 16:495-505.

Natarajan, B., Kalsi, H. S., Godbole, P., Malankar, N., Thiagarayaselvam, A., Siddappa, S., Thulasiram, H. V., Chakrabarti, S. K., and Banerjee, A. K. 2018. MiRNA160 is associated with local defense and systemic acquired resistance against Phytophthora infestans infection in potato. J. Exp. Bot. 69:2023-2036.

Neill, S. J., Desikan, R., Clarke, A., Hurst, R. D., and Hancock, J. T. 2002. Hydrogen peroxide and nitric oxide as signalling molecules in plants. J. Exp. Bot. 53:1237-1247.

Pei, L., Zhang, L., Li, J., Shen, C., Qiu, P., Tu, L., Zhang, X., and Wang, M. 2019. Tracing the origin and evolution history of methylation-related genes in plants. BMC Plant Biol. 19:307.

Piya, S., Bennett, M., Rambani, A., and Hewezi, T. 2017a. Transcriptional activity of transposable elements may contribute to gene expression changes in the syncytium formed by cyst nematode in Arabidopsis roots. Plant Signal. Behav. 12:e1362521.

Piya, S., Kihm, C., Rice, J. H., Baum, T. J., and Hewezi, T. 2017b. Cooperative regulatory functions of miR858 and MYB83 during cyst nematode parasitism. Plant Physiol. 174:1897-1912.

Quinlan, A. R., and Hall, I. M. 2010. BEDTools: A flexible suite of utilities for comparing genomic features. Bioinformatics 26:841-842.

Rambani, A., Pantalone, V., Yang, S., Rice, J. H., Song, Q., Mazarei, M. Arelli, P. R., Meksem, K., Stewart, C. N., and Hewezi, T. 2020. Identification of introduced and stably inherited DNA methylation variants in soybean associated with soybean cyst nematode parasitism. New Phytol. 227:168-184.

Rambani, A., Rice, J. H., Liu, J., Lane, T., Ranjan, P., Mazarei, M., Pantalone, V., Stewart, C. N., Jr., Staton, M., and Hewezi, T. 2015. The methylome of soybean roots during the compatible interaction with the soybean cyst nematode. Plant Physiol. 168:1364-1377.

Rogers, K., and Chen, X. 2013. Biogenesis, turnover, and mode of action of plant microRNAs. Plant Cell 25:2383-2399.

Secco, D., Wang, C., Shou, H., Schultz, M. D., Chiarenza, S., Nussaume, L., Ecker, J. R., Whelan, J., and Lister, R. 2015. Stress induced gene expression drives transient DNA methylation changes at adjacent repetitive elements. eLife 4:e09343. 
Shamimuzzaman, M., and Vodkin, L. 2012. Identification of soybean seed developmental stage-specific and tissue-specific miRNA targets by degradome sequencing. BMC Genomics 13:310.

Shayevitch, R., Askayo, D., Keydar, I., and Ast, G. 2018. The importance of DNA methylation of exons on alternative splicing. RNA 24:1351-1362.

Shen, J., Wang, S., Zhang, Y. J., Kappil, M. A., Chen Wu, H., Kibriya, M. G., Wang, Q., Jasmine, F., Ahsan, H., Lee, P. H., Yu, M. W., Chen, C. J., and Santella, R. M. 2012. Genome-wide aberrant DNA methylation of microRNA host genes in hepatocellular carcinoma. Epigenetics 7:1230-1237.

Siddique, S., Matera, C., Radakovic, Z. S., Hasan, M. S., Gutbrod, P., Rozanska, E., Sobczak, M., Torres, M. A., and Grundler, F. M. 2014. Parasitic worms stimulate host NADPH oxidases to produce reactive oxygen species that limit plant cell death and promote infection. Sci. Signal. 7:ra33.

Smirnoff, N., and Arnaud, D. 2019. Hydrogen peroxide metabolism and functions in plants. New Phytol. 221:1197-1214.

Song, Q.-X., Liu, Y.-F., Hu, X.-Y., Zhang, W.-K., Ma, B., Chen, S.-Y., and Zhang, J.-S. 2011. Identification of miRNAs and their target genes in developing soybean seeds by deep sequencing. BMC Plant Biol. 11:5.

Song, Y., Ci, D., Tian, M., and Zhang, D. 2016. Stable methylation of a noncoding RNA gene regulates gene expression in response to abiotic stress in Populus simonii. J. Exp. Bot. 67:1477-1492.

Song, Y., Tian, M., Ci, D., and Zhang, D. 2015. Methylation of microRNA genes regulates gene expression in bisexual flower development in andromonoecious poplar. J. Exp. Bot. 66:1891-1905.

Supek, F., Bošnjak, M., Skunca, N., and Šmuc, T. 2011. REVIGO summarizes and visualizes long lists of gene ontology terms. PLoS One 6:e21800.

Suzuki, M. M., and Bird, A. 2008. DNA methylation landscapes: Provocative insights from epigenomics. Nat. Rev. Genet. 9:465-476.

Tian, B., Wang, S., Todd, T. C., Johnson, C. D., Tang, G., and Trick, H. N. 2017. Genome-wide identification of soybean microRNA responsive to soybean cyst nematodes infection by deep sequencing. BMC Genomics 18:572.

Varghese, V. K., Shukla, V., Kabekkodu, S. P., Pandey, D., and Satyamoorthy, K. 2018. DNA methylation regulated microRNAs in human cervical cancer. Mol. Carcinog. 57:370-382.

Verwoerd, T. C., Dekker, B. M., and Hoekema, A. 1989. A small-scale procedure for the rapid isolation of plant RNAs. Nucleic Acids Res. 17: 2362.

Wan, J., Vuong, T., Jiao, Y., Joshi, T., Zhang, H., Xu, D., and Nguyen, H. T. 2015. Whole-genome gene expression profiling revealed genes and pathways potentially involved in regulating interactions of soybean with cyst nematode (Heterodera glycines Ichinohe). BMC Genomics 16:148.

Wang, X., Weigel, D., and Smith, L. M. 2013. Transposon variants and their effects on gene expression in Arabidopsis. PLoS Genet. 9:e1003255.

Wibowo, A., Becker, C., Marconi, G., Durr, J., Price, J., Hagmann, J., Papareddy, R., Putra, H., Kageyama, J., Becker, J., Weigel, D., and Gutierrez-
Marcos, J. 2016. Hyperosmotic stress memory in Arabidopsis is mediated by distinct epigenetically labile sites in the genome and is restricted in the male germline by DNA glycosylase activity. eLife 5:e13546.

Wong, J., Gao, L., Yang, Y., Zhai, J., Arikit, S., Yu, Y., Duan, S., Chan, V., Xiong, Q., Yan, J., Li, S., Liu, R., Wang, Y., Tang, G., Meyers, B. C., Chen, X., and Ma, W. 2014. Roles of small RNAs in soybean defense against Phytophthora sojae infection. Plant J. 79:928-940.

Xu, M., Li, Y., Zhang, Q., Xu, T., Qiu, L., Fan, Y., and Wang, L. 2014. Novel miRNA and phasiRNA biogenesis networks in soybean roots from two sister lines that are resistant and susceptible to SCN race 4. PLoS One 9:e110051.

Xu, S., Liu, N., Mao, W., Hu, Q., Wang, G., and Gong, Y. 2016. Identification of chilling-responsive microRNAs and their targets in vegetable soybean (Glycine max L.). Sci. Rep. 6:26619.

Yin, Y., Morgunova, E., Jolma, A., Kaasinen, E., Sahu, B., Khund-Sayeed, S., Das, P. K., Kivioja, T., Dave, K., Zhong, F. and Nitta, K. R. 2017. Impact of cytosine methylation on DNA binding specificities of human transcription factors. Science 356:eaaj2239.

Yong-Villalobos, L., González-Morales, S. I., Wrobel, K., GutiérrezAlanis, D., Cervantes-Peréz, S. A., Hayano-Kanashiro, C., OropezaAburto, A., Cruz-Ramírez, A., Martínez, O., and Herrera-Estrella, L. 2015. Methylome analysis reveals an important role for epigenetic changes in the regulation of the Arabidopsis response to phosphate starvation. Proc. Natl. Acad. Sci. U.S.A. 112:E7293-E7302.

Yu, B., Yang, Z., Li, J., Minakhina, S., Yang, M., Padgett, R. W., Steward, R., and Chen, X. 2005. Methylation as a crucial step in plant microRNA biogenesis. Science 307:932-935.

Zhang, C., Ding, Z., Wu, K., Yang, L., Li, Y., Yang, Z., Shi, S., Liu, X., Zhao, S., Yang, Z., Wang, Y., Zheng, L., Wei, J., Du, Z., Zhang, A., Miao, H., Li, Y., Wu, Z., and Wu, J. 2016. Suppression of jasmonic acidmediated defense by viral-inducible microRNA319 facilitates virus infection in rice. Mol. Plant 9:1302-1314.

Zhang, H., Lang, Z., and Zhu, J. K. 2018. Dynamics and function of DNA methylation in plants. Nat. Rev. Mol. Cell Biol. 19:489-506.

Zhao, M., Cai, C., Zhai, J., Lin, F., Li, L., Shreve, J., Thimmapuram, J., Hughes, T. J., Meyers, B. C., and Ma, J. 2015. Coordination of microRNAs, phasiRNAs, and NB-LRR Genes in response to a plant pathogen: Insights from analyses of a set of soybean Rps gene nearisogenic lines. Plant Genome 8:1-13.

Zhao, Q., Sun, C., Liu, D. D., Hao, Y. J., and You, C. X. 2015. Ectopic expression of the apple Md-miR172e gene alters flowering time and floral organ identity in Arabidopsis. Plant Cell Tiss. Org. 123: 535-546.

Zhao, W., Li, Z., Fan, J., Hu, C., Yang, R., Qi, X., Chen, H., Zhao, F., and Wang, S. 2015. Identification of jasmonic acid-associated microRNAs and characterization of the regulatory roles of the miR319/TCP4 module under root-knot nematode stress in tomato. J. Exp. Bot. 66:4653-4667. 